\title{
INFLUENCE OF TESTING METHODOLOGY ON POSITION OF THE FORMING LIMIT CURVE
}

\author{
1'Jiří SOBOTKA, ${ }^{1}$ Pavel SOLFRONK, 'David KOREČEK, ${ }^{1}$ Petr PILAŘ \\ ${ }^{1} T U L$ - Technical University of Liberec, Liberec, Czech Republic, EU, \\ jiri.sobotka@tul.cz, pavel.solfonk@tul.cz, david.korecek@tul.cz, petr.pilar@tul.cz
}

https://doi.org/10.37904/metal.2020.3474

\begin{abstract}
Determining the limit states (both theoretically and experimentally) of formed parts in the sheet metal forming represents quite important material characteristics, which are commonly applied e.g. in the field of numerical simulations. Within the precise determination of the limit state, there is not used only a relatively large number of theoretical approaches (e.g. theoretical Keeler-Goodwin diagram or MK theory), but also various experimental approaches. Thus, in addition to the basically preferred approach (the so-called Nakazima test), other experimental methods are commonly used - e.g. stretching shaped workpieces by the hemispherical punch and circular die, tensile test of specimens with different notches, common hydrostatic bulge test with circular or elliptical die or e.g. utilization of punches with different radii. Two different experimental approaches were used in this paper to determine limit state - to be specific position of the forming limit curve (FLC) in the forming limit diagram (FLD) for tested deep-drawing material DX57D. The contact-less optical system MERCURY RT from the company Sobriety was used to measure the distribution of true strains (major and minor one). In the experimental part, methods of stretching shaped workpieces by the hemispherical punch and circular die (can be applied to both parts of the FLC) and tensile test of samples provided with different notches (this one can be applied only to the left part of the FLC) were used. From the values of final limit states in light of strain and their mutual comparison, it is possible to deduce the influence of the chosen methodology (results from the first experimental approach are taken as a reference state) and thus also their possible applicability and reliably in the experimental determination of FLC's.
\end{abstract}

Keywords: Forming limit curve, sheet metal forming, deep-drawing material, contact-less optical deformation measurement, photogrammetry

\section{INTRODUCTION}

Within the sheet metal forming is knowledge of the materials limit states truly very important, because it provides information both to constructer and technologist about maximal achievable strains of applied material under different stress states that can occur at processing sheets. And as a boundary between safe conditions and failure of material, it serves so-called forming limit curve (FLC). A plot of the combination of strains that lead to failure is subsequently called a forming limit diagram (FLD) [1]. During the last decades, quite a lot of theoretical and experimental approaches have been investigated and verified in this area [2, 3]. Regarding such numerous approaches, there is always issue about their accuracy and reliability. As a "conventional" standard is used so-called Nakazima test, i.e. drawing rectangular samples having different widths by hemispherical punch and circular die [4,5]. Similar experimental method was also used in this paper at first.

In addition to that, there has been recently found an increasing application of so-called hybrid experimental methods, which determine the forming limit curve (FLC) only from the measurement of the static tensile test and relevant anisotropy coefficients. These methods are thus used in the context of fast and approximate determination of the forming limit curve position in the forming limit diagram (FLD), when the equipment for its precise determination by classical methods is not available. 


\section{METHODOLOGICAL BASES - TESTING MATERIAL AND “CONVENTIONAL” APPROACH}

As a tested material there was chosen the deep-drawing material DX57D Z100 MBO of thickness $0.6 \mathrm{~mm}$. It is low-carbon steel that is mainly used in the automotive industry for the car-body outer panels. Basic mechanical properties (measured from 10 samples) of this material are summarized in Table 1.

Table 1 Basic mechanical properties of tested deep-drawing material DX57D Z100 MBO

\begin{tabular}{|c|c|c|c|c|c|}
\hline \multicolumn{2}{|c|}{$\begin{array}{l}\text { Basic mechanical properties } \\
\quad\left(\text { rolling direction } 0^{\circ}\right)\end{array}$} & $\begin{array}{l}\text { Proof yield } \\
\text { strength } \\
R_{p 02}(\mathrm{MPa})\end{array}$ & $\begin{array}{l}\text { Ultimate tensile } \\
\text { strength } \\
\quad \boldsymbol{R}_{\boldsymbol{m}}(\mathrm{MPa})\end{array}$ & $\begin{array}{c}\text { Uniform } \\
\text { ductility } \\
\boldsymbol{A}_{g}(\%)\end{array}$ & $\begin{array}{c}\text { Total } \\
\text { ductility } \\
\text { A80mm (\%) }\end{array}$ \\
\hline \multirow{2}{*}{ DX57D } & arithmetic mean $\boldsymbol{x}$ & 154.3 & 295.5 & 26.12 & 45.28 \\
\hline & standard deviation $\boldsymbol{s}$ & 1.2 & 2.1 & 0.42 & 0.35 \\
\hline
\end{tabular}

As there was already mentioned before, the major aim of this paper was to compare relevant part of FLC regarding the applied measuring procedure. The first one represents the "conventional" approach (very close to the classical Nakazima test), which used hemispherical punch and circular die to stretch shaped workpieces. Change of the stress state is there achieved by changing their geometry, where varies the minimal width (the central one) between curved parts, whose radius is always $105 \mathrm{~mm}$. Magnitude of such width was as following: $30,45,60,90,105,120,130,140,160,180$ and finally $210 \mathrm{~mm}$. Thus there were used 11 different geometries that are generally marked according to their width (e.g. FLD 30 or FLD 90 for samples having such central width of $30 \mathrm{~mm}$ or $90 \mathrm{~mm}$; "full" samples are subsequently marked as FLD 210 - see Figure 1). Final FLD of tested DX57D steel (determined by that "conventional" measurement) is shown in Figure 1. Stress state is changing from the uniaxial tensile stress state (very close to the left boundary line - static tensile test) to the equi bi-axial stretching (next to the right boundary line) as it is also shown by black lines in Figure 1.

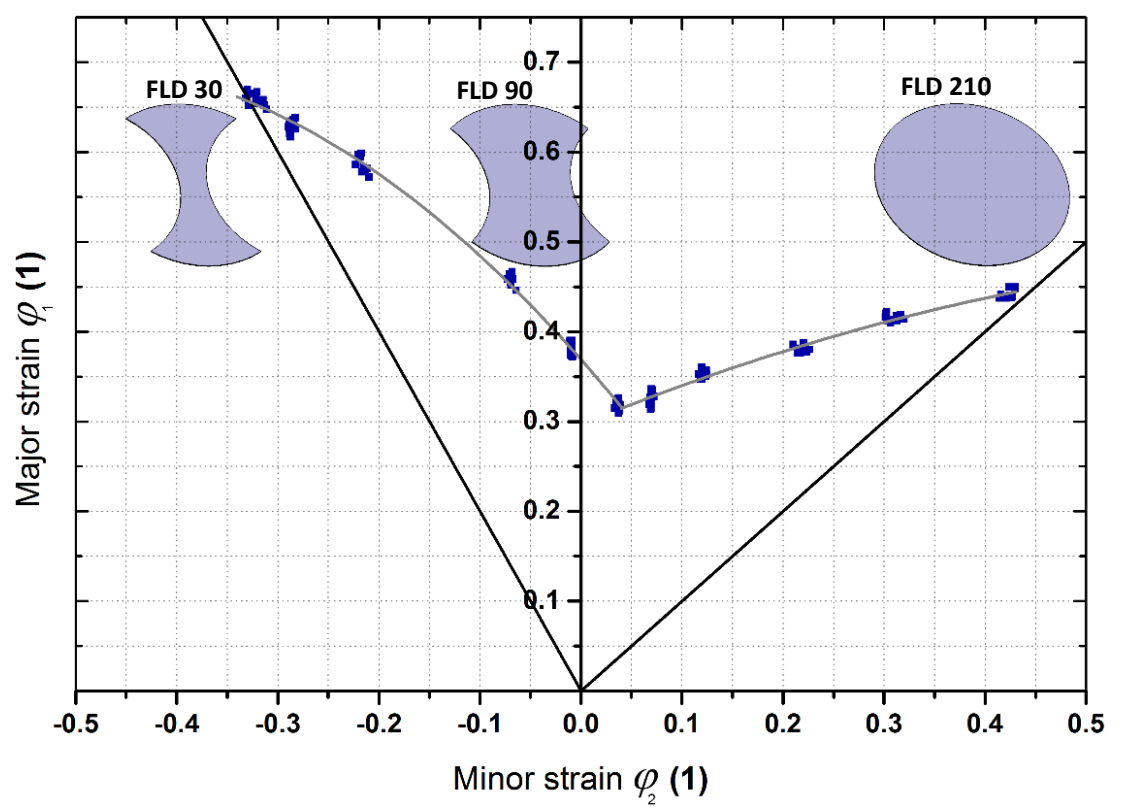

Figure 1 FLD of tested material DX57D with examples of samples geometry (FLD 30, 90 and 210)

\section{DIFFERENT EXPERIMENTAL APPROACH AND RESULTS}

Beside "conventional" determination of FLD for tested material, there were also prepared notched samples to determine the influence of these notches on the position of FLC. In Figure 2 is shown testing position of sample 
marked like NOTCH 1 (left) and environment of testing software WorkBench II (right) that is used to control the whole newly developed planar bi-axial testing machine (see Figure 3).
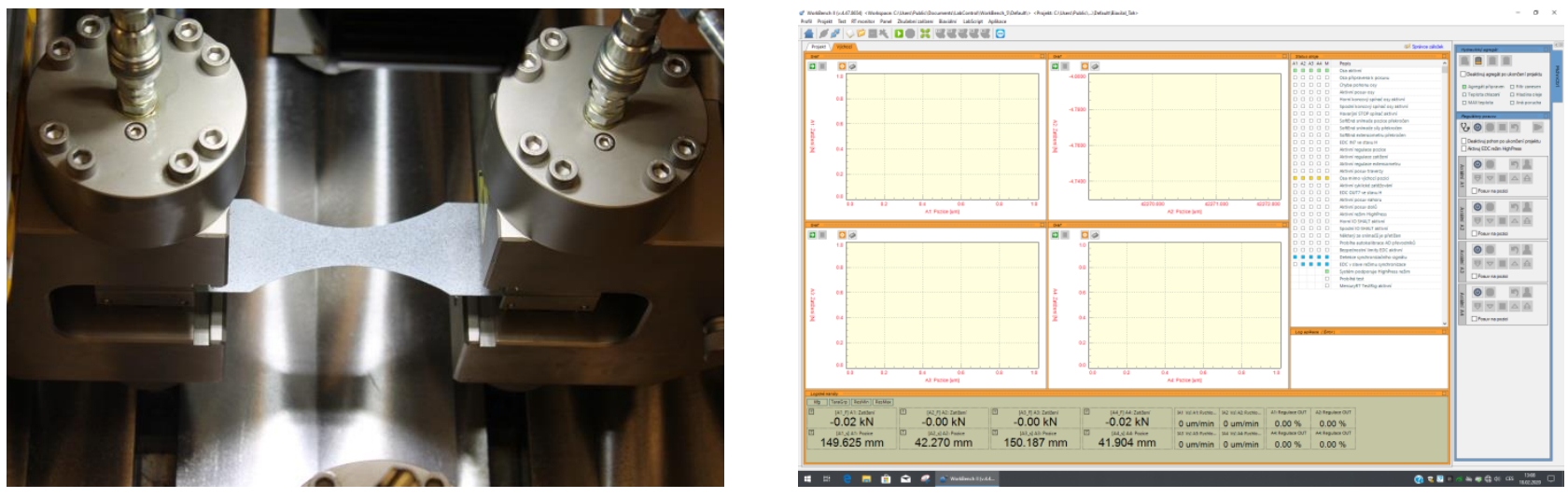

Figure 2 Position of sample NOTCH 1 (left) and working environment of software WorkBech II (right)

At the Department of Engineering Technology (TU of Liberec) was designed the new planar bi-axial testing machine (detail of own measuring unit is shown in Figure 3) that generally consists of two pairs of cylinders (thus totally four cylinders), which deformed tested specimen under required different stress and strain states. In this case was just used the uniaxial tensile stress state - so only two cylinders were active. In addition to that, there were applied also optical cameras and lighting for contact-less deformation measurement by the system MERCURY RT. The whole testing was carried out at room temperature and strain rate $c=0.002 \mathrm{~s}^{-1}$.

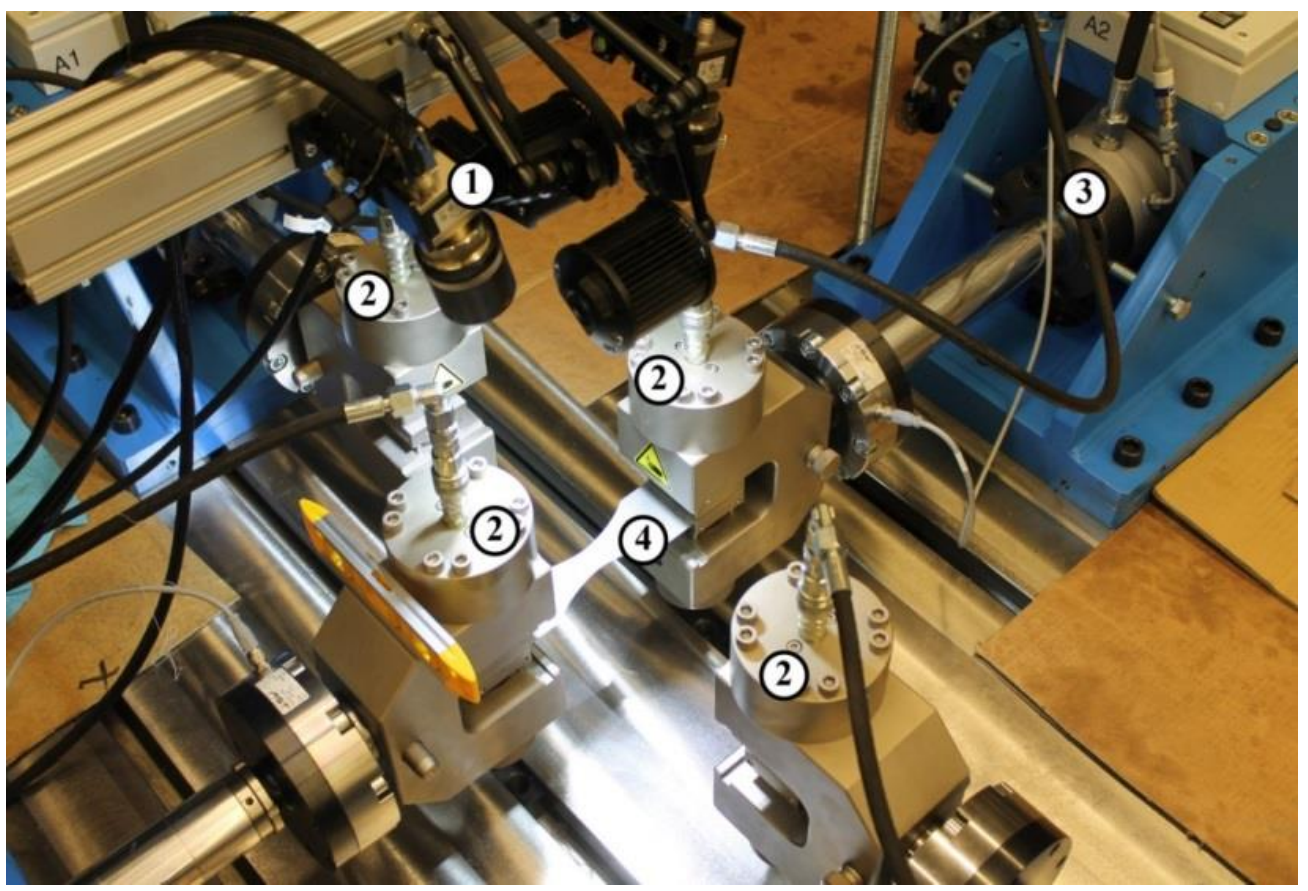

Figure 3 Detail of planar bi-axial testing machine

(1 - contact-less optical measurement, 2 - hydraulic clamping, 3 - hydraulic cylinder, 4 - tested sample)

The chosen geometry of notched samples is schematically shown in Figure 4. Totally, there were prepared six different geometries marked as NOTCH $1-6$. The minimal width (the central one) of all samples was $30 \mathrm{~mm}$ and used radii were as following: NOTCH $1-\mathrm{R} 100,2-\mathrm{R} 75,3-\mathrm{R} 50,4-\mathrm{R} 25,5-\mathrm{R} 15$ and $6-\mathrm{R} 7.5$. Initial dimensions (length $\mathrm{x}$ width) of these samples were $300 \times 65 \mathrm{~mm}$. 


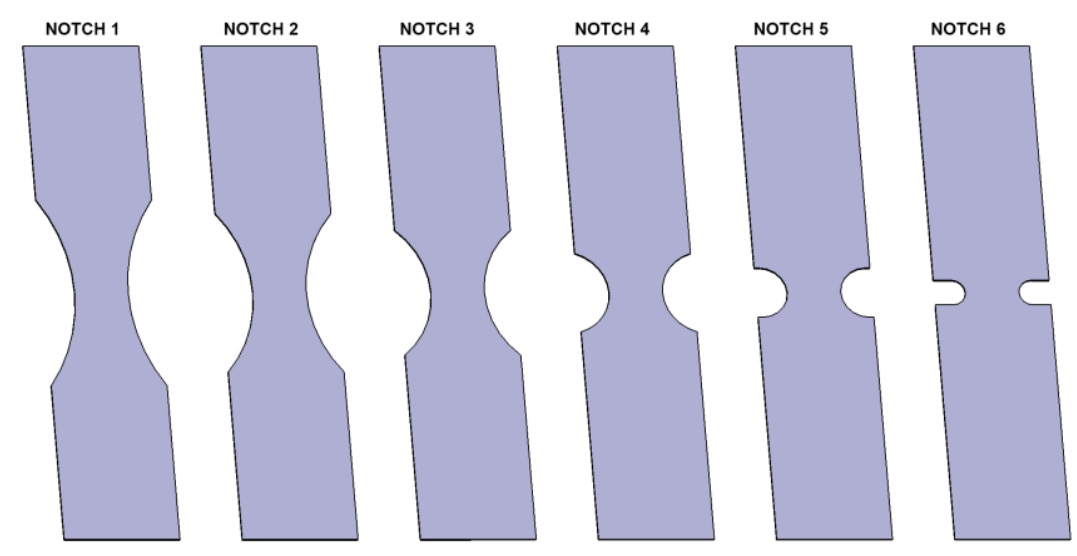

Figure 4 Geometry of the notched samples

Also in this case was used contact-less optical deformation measurement via system MERCURY RT. Examples of evaluated images (right before fracture and at data scanning frequency of $12 \mathrm{fps}$ ) with distribution of major strain $\varphi_{1}$ (1) and applied sections are shown in Figure 5. On the left is displayed NOTCH 1 (very similar to the static tensile test - sample no. 3) and on the right is shown NOTCH 5 (sample no. 3).

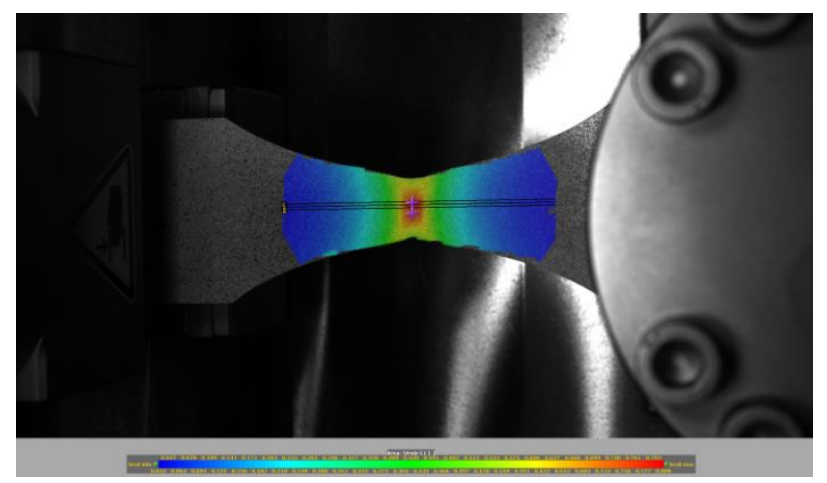

Figure 5 Distribution of major strain $\varphi_{1}$ (1) for NOTCH 1 (left) and NOTCH 5 (right)

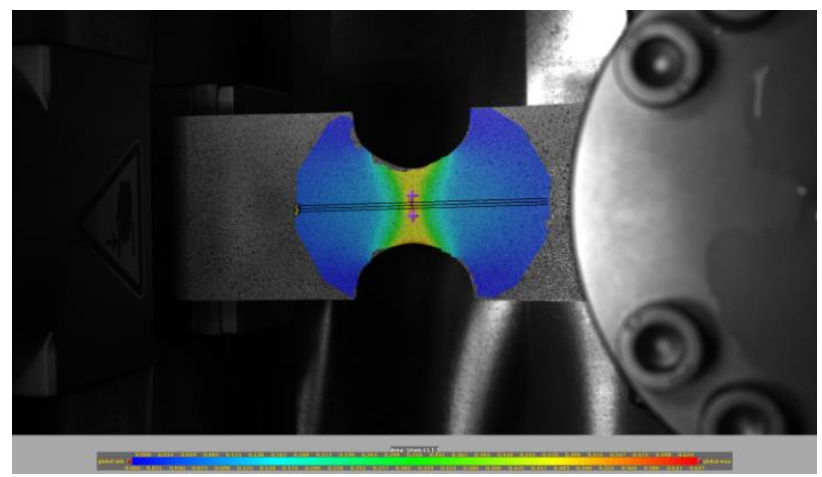

Figure 6 illustrates the necessary condition that has to be always done during evaluation of the forming limit diagrams acc. to standard ISO/DIS 12004-2. Here can't be used maximal values, but regarding the localization of deformation (necking) there has to be applied parabolas open downward whose vertexes as finally taken as results. The same approach was also used at measurement the "conventional" FLC of tested material.

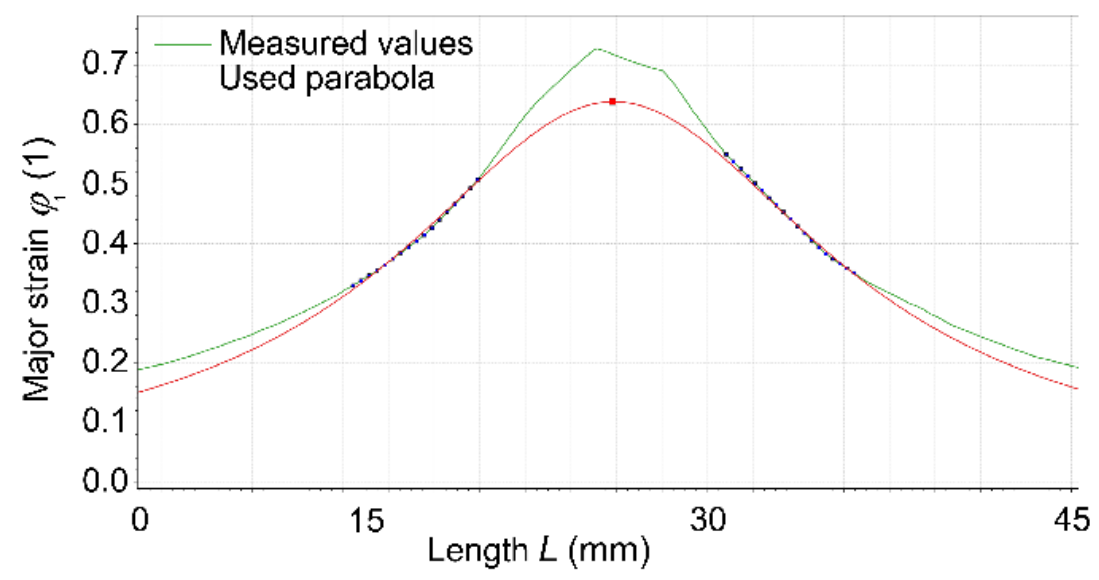

Figure 6 Example of application parabola at finding results of FLC for NOTCH 1 
The most important graphical comparison is shown in Figure 7, where are compared "conventional" samples (black dots and line) and notched samples (olive dots and dashed line) both in the form of scatters and as FLC curves. As there was already mentioned before, because of notches samples is there shown only left part of FLD - see values on X-axis. Moreover, there is also added the left limit of FLD's as line having slope -2 . Sometimes are used samples already designed for static tensile test in the case of samples marked here as FLD 30 or NOTCH 1, because stress state in this case is the uniaxial tensile one.

Quite interesting tendency can be observed from the measured results, which can be divided into two basic groups. Among the first group can be found the notched samples marked like NOTCH 1 (R100), NOTCH 2 (R75) and NOTCH 3 (R50) that reveal almost the same results as "conventional" ones with the lowest width (FLD 30 and FLD 45). In addition to the performed experiments, there were also tested static tensile samples and results of these tests (not shown in Figure 7) could be also find in the area of final values for samples FLD 30 as well as for NOTCH 1 . On the other hand, already in this area it can be observed the tendency of faster decrease (olive line) for notched samples then in the case of "conventional" ones.

The second group consists of notched samples marked like NOTCH 4 (R25), NOTCH 5 (R15) and at the end NOTCH 6 (R7.5). In this area is difference between FLD's samples (FLD 60, FLD 90 and FLD 105) and notched samples still more and more evident. The higher magnitude of minor strain $\varphi_{2}(1)$, the higher difference between two experimental approaches. Thus the maximal difference was observed in the case of NOTCH 6 and its relevant position of FLC measured "conventionally". It is evident that influence of the notch increases with the decreasing magnitude of used radius (in this case from R100 to R7.5). As the geometry of the notched sample becomes in light of radius more "severe" (smaller values of radius), there is faster achieving of the tested material limit state due to earlier localization of deformation and fracture of sample.

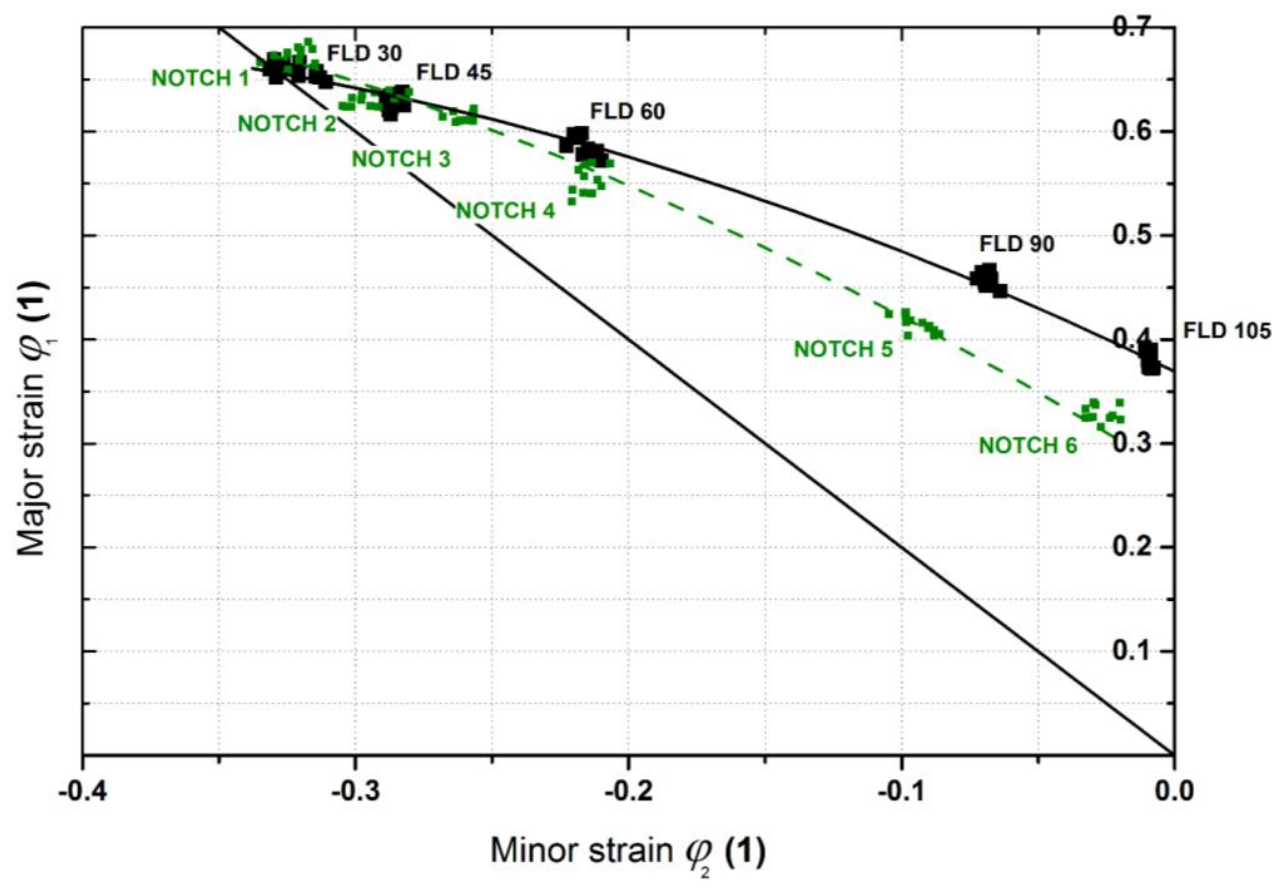

Figure 7 Graphical comparison between "conventional" and "notched" samples of the FLC left part

\section{CONCLUSION}

The major aim of this paper was to compare (in the relevant area) two different experimental approaches, which can be generally used at determination the forming limit curve (FLC) in the forming limit diagram (FLD) of metal sheets. As a tested material was chosen the deep-drawing material marked as DX57 D Z100 MBO of thickness $0.6 \mathrm{~mm}$. It is zinc-coated sheet that is mainly used for car-body outer panels in the automotive. 
The first experimental method was performed by the hemispherical punch to stretch shaped workpieces. In this case, the stress state is changed by different geometry of samples (i.e. different minimal width in the centre of sample - see chapter 2). There were totally used 11 different geometries of testing samples (width was as following: $30,45,60,90,105,120,130,140,160,180$ and finally "full" samples having diameter of $210 \mathrm{~mm}$ ). FLC measured by this method covers the full range within FLD and is shown, together with the examples of samples geometry, in Figure 1. The second experimental approach consisted of utilization different geometries of notches at uniaxial loading of these samples. Totally, there were used six geometries of notches in the experimental part. Schematically are these samples shown in Figure 4 and their designation was as following: NOTCH 1 - R100, NOTCH 2 - R75, NOTCH 3 - R50, NOTCH 4 - R25, NOTCH 5 - R15 and NOTCH 6 R7.5. However, FLC measured by this method cover only left part within FLD, so the final comparison of these two methods was also done just in this region.

The final comparison from the graphical point of view is given in Figure 7, where is shown the left part of FLD for both applied experimental approaches ("conventional" method is black and "notch" approach in olive). From such comparison is there obvious the following tendency - the higher magnitude of minor strain $\varphi_{2}(1)$, the higher difference between two experimental methods. Regarding the geometry of notched samples, there is evident that as notch becomes more and more "severe", there is earlier localization of deformation. It results in the failure of sample already before achieving its truly limit state. Based upon these results, there can be stated that (at least for tested deep-drawing material DX57D) is the second used experimental method (notched one) not very favourable and should not be used for determination left part of relevant FLC. On the other hand, these are just the first results and in addition to that only for one material. Such conclusion should be verified by further research in this branch, including other thicknesses of deep-drawing materials or other types of materials (e.g. high-strength steels or aluminium alloys). There can be also used other methods for FLC determination (e.g. utilization of punches with different radii) as well.

\section{ACKNOWLEDGEMENTS}

\section{This work was supported by the Student Grant Competition of the Technical University of Liberec under the project No. SGS-2019-5015 "Research and development for innovation of materials and production technologies with application potential in mechanical engineering".}

\section{REFERENCES}

[1] HOSFORD, William F., CADDEL, M. Robert. Metal Forming-Mechanics and Metallurgy. 3rd ed. Cambridge: Cambridge University Press, 2007.

[2] WANKHEDE, P., SURESH, K. A review on the evaluation of formability in sheet metal forming. Advances in Materials and Processing Technologies. 2020, vol. 6, no. 2, pp.458-485.

[3] BASAK, S., PANDA S.K., LEE M.-G. Formability and fracture in deep drawing sheet metals: Extended studies for pre-strained anisotropic thin sheets. International Journal of Mechanical Sciences. 2020, vol. 170, 105346.

[4] KUHN, H., MEDLIN D., ed. ASM HANDBOOK Volume 8 - Mechanical Testing and Evaluation. 10th ed. Materials Park, Russell Township, OH: ASM International, 2000. ISBN 0-87170-389-0.

[5] ASHBY, M.F. Materials Selection in Mechanical Design. 3rd ed. Oxford: Butterworth-Heinemann, 2005. ISBN 07506-6168-2. 